\title{
Weak lower semicontinuity for non coercive polyconvex integrals
}

\author{
Micol Amar, Virginia De Cicco, Paolo Marcellini and Elvira Mascolo \\ Communicated by N. Fusco
}

\begin{abstract}
We prove a lower semicontinuity theorem for a polyconvex functional of integral form, related to maps $u: \Omega \subset \mathbb{R}^{n} \rightarrow \mathbb{R}^{m}$ in $W^{1, n}\left(\Omega ; \mathbb{R}^{m}\right)$ with $n \geq m \geq 2$, with respect to the weak $W^{1, p}$-convergence for $p>m-1$, without assuming any coercivity condition.
\end{abstract}

Keywords. Vector-valued maps, Jacobian determinants, polyconvex integrals, lower semicontinuity, chain rule.

AMS classification. 49J45, 49K20.

\section{Introduction}

Direct methods of calculus of variations are a rigorous framework to treat mathematically interesting problems arising in science or engineering related to optimality conditions such as the minimization of the energy (or minimization of time, or of space, etc.), expressed in integral form such as, for instance,

$$
\int_{\Omega} g(x, u, D u) d x .
$$

The mathematical theory of direct methods, rougly speaking, is based on the lower semicontinuity of the energy functional in (1.1) in a certain topology and on the relative compactness of bounded sets of functions $u$ which compete for a minimum. These two properties are the main ingredients in order to assure the existence of equilibrium solutions. Compactness has been a main motivation for the introduction of theory of Functional Analysis in the last century. Lower semicontinuity, either in $L^{p}$ or in the weak topology of $W^{1, p}$ (the Sobolev space of $L^{p}$-functions with first partial derivatives in $L^{p}$ ), classically is based on the convexity of the function $g$ in (1.1) with respect to the gradient variable $D u$. However, motivated by the applications to nonlinear elasticity, in $1977 \mathrm{~J}$. Ball pointed out in [4] that convexity is completely unrealistic in the vectorial case. Indeed, it conflicts, for instance, with the natural requirement that the elastic energy is frame-indifferent. Hence, it must be replaced by different and

The present work has been partially supported by GNAMPA-INdAM (Gruppo Nazionale per l'Analisi Funzionale e Applicazioni-Istituto Nazionale di Alta Matematica), project "Singolarità e semicontinuità nel Calcolo delle Variazioni”. 
more general conditions, named quasiconvexity and polyconvexity, properties already introduced theoretically by Morrey in [25]. These new convexity conditions are now considered fundamental ones in nonlinear elasticity. In particular, polyconvexity takes into account a natural constitutive hypothesis which is the invariance of the energy under the transformation $g \mapsto g+\phi$, for every null Lagrangian $\phi$.

The aim of this research is to consider the polyconvexity condition, to achieve new lower semicontinuity results useful for applications in this field. We describe below in more details the mathematical problem.

Dealing with vector-valued maps, still it is not completely known a set of minimal assumptions for lower semicontinuity of integrals of the Calculus of Variations of the form

$$
d(u)=\int_{\Omega} g(x, u, D u) d x,
$$

where $u: \Omega \rightarrow \mathbb{R}^{m}$ is a vector-valued map defined in an open set $\Omega \subset \mathbb{R}^{n}$ and $D u$ is the $m \times n$ Jacobian matrix of its partial derivatives

$$
u \equiv\left(u^{1}, u^{2}, \ldots, u^{m}\right), \quad D u=\left(\frac{\partial u^{\alpha}}{\partial x_{i}}\right)_{i=1,2, \ldots, n}^{\alpha=1,2, \ldots, m} .
$$

On the contrary, in the so-called scalar case (corresponding to $m=1$ ) Serrin in 1961, in the pioneering paper [27], pointed out the convexity of $g=g(x, s, \xi)$ with respect to the gradient variable $\xi$ as a main (necessary and) sufficient condition for the lower semicontinuity of the integral $d(u)$ in (1.2). Serrin himself pointed out the special role of the $x$ variable to obtain the lower semicontinuity of $d(u)$ with respect to the $L_{\text {loc }}^{1}(\Omega)$-convergence. In fact, among his assumptions we mention either the coercivity of $g$, i.e.,

$$
g(x, s, \xi) \geq c|\xi|
$$

for some positive constant $c$ and for every $(x, s, \xi) \in \Omega \times \mathbb{R} \times \mathbb{R}^{n}$, or the continuity of the partial derivatives

$$
g_{x}(x, s, \xi), \quad g_{\xi}(x, s, \xi), \quad g_{\xi x}(x, s, \xi) .
$$

Some extensions of Serrin's results have been recently obtained by Gori-Marcellini [19] and by several other authors, also in the context of $B V$-functions (see $[18,15,9$, $10,3])$.

In the vector-valued case $m>1$ either the quasiconvexity or the polyconvexity of $g$ with respect to the gradient variable $\xi$ play a role. These convexity conditions are due to Morrey [25]; a reference paper is Ball [4]. In particular the function $g(x, s, \xi)$ is said polyconvex with respect to the gradient variable $\xi$ if it can be represented under the form

$$
g(x, s, \xi)=f(x, s, M(\xi)),
$$


where $f$ is a convex function with respect to its last variable and, for every $m \times n$ matrix $\xi \in \mathbb{M}^{m \times n}, M(\xi)$ denotes the vector

$$
M(\xi)=\left(\xi, \operatorname{adj}_{2} \xi, \ldots, \operatorname{adj}_{i} \xi, \ldots, \operatorname{adj}_{\min \{m, n\}} \xi\right) .
$$

Here $\operatorname{adj}_{i} \xi$, for $i=2, \ldots, \min \{m, n\}$, stands as the vector of the determinants of the $i \times i$ minors of the matrix $\xi \in \mathbb{M}^{m \times n}$. Thus $M(\xi)$ is a vector in $\mathbb{R}^{\tau}$, with

$$
\tau=\tau(n, m)=\sum_{i=1}^{\min (m, n)}\left(\begin{array}{l}
n \\
i
\end{array}\right)\left(\begin{array}{c}
m \\
i
\end{array}\right) .
$$

We assume that $f: \Omega \times \mathbb{R}^{m} \times \mathbb{R}^{\tau} \rightarrow[0,+\infty)$ is a nonnegative function, convex in $\mathbb{R}^{\tau}$ with respect to the last variable.

The lower semicontinuity for polyconvex integrals have been investigated by several authors in the past years, starting from the results by Morrey and Ball cited above. In the quoted papers the weak topology of $W^{1, p}\left(\Omega ; \mathbb{R}^{m}\right)$ is considered, for some $p>\min \{m, n\}$. A more recent counterexample by Maly [22] shows that the semicontinuity in the weak topology of $W^{1, p}\left(\Omega ; \mathbb{R}^{m}\right)$ is generically not true if $p<\min \{m, n\}-1$. Marcellini [23, 24] and Dacorogna-Marcellini [7] proved a lower semicontinuity result when $p>n-1, m=n$ and $f=f(M(\xi))$ or $f=f(x, M(\xi))$. The limit case $p=n-1$ has been studied in the same context by Acerbi-Dal Maso [2], Dal Maso-Sbordone [8], Celada-Dal Maso [5] and FuscoHutchinson [16]. See also Acerbi-Buttazzo-Fusco [1] for lower semicontinuity with respect to the $L^{\infty}$-topology.

The situation changes considerably when a dependence on $s$ is also allowed, since the presence of the $(x, s)$-variables cannot be treated as a simple perturbation. A result in this more general context is due to Gangbo [17] under the following structure assumptions: $f(x, s, M(\xi))=a(x, s) h(x, M(\xi))$. More recently Fonseca-Leoni [14], by using a blow-up argument, proved a semicontinuity result under the coercivity condition

$$
f(x, s, M(\xi)) \geq c|M(\xi)|,
$$

valid for some positive constant $c$ and for every $(x, s, \xi) \in \Omega \times \mathbb{R}^{m} \times \mathbb{R}^{m \times n}$. They consider the weak topology of $W^{1, p}\left(\Omega ; \mathbb{R}^{n}\right)$ in the limit case $p=n-1$ too, with $f$ depending only on the Jacobian determinant $\operatorname{det} \xi=\operatorname{adj}_{n} \xi$ of the $n \times n$ matrix $\xi$, i.e., $f=f(x, s, \operatorname{det} \xi)$ and the coercivity condition $f(x, s, \operatorname{det} \xi) \geq c|\operatorname{det} \xi|$ holds.

In the spirit of Serrin's work, where either the coercivity or the continuity of the partial derivatives in (1.3) were assumed, in this paper we consider the vector-valued case $m>1$ and we study the lower semicontinuity of the integral in (1.2) related to general polyconvex integrands; the lack of coercivity and the general form of the dependence on the $(x, s)$-variables force additional regularity assumptions on the integrand. In particular we prove the following result (for more general assumptions see Theorems 4.2 and 4.3). 
Theorem. Let us consider the vector-valued case $m \geq 2$ and let $n \geq m$. Let $f$ be a nonnegative convex function with respect to the last variable, such that $f$ and $f_{x}$ are continuous in $\Omega \times \mathbb{R}^{m} \times \mathbb{R}^{\tau}$. Then, for every $u_{k}, u \in W^{1, m}\left(\Omega, \mathbb{R}^{m}\right)$, such that $u_{k}$ converges to $u$ in the weak topology of $W^{1, p}\left(\Omega, \mathbb{R}^{m}\right)$ for some $p>m-1$, we have

$$
\liminf _{k \rightarrow+\infty} \int_{\Omega} f\left(x, u_{k}, M\left(D u_{k}\right)\right) d x \geq \int_{\Omega} f(x, u, M(D u)) d x .
$$

We explicitly note that the condition $u_{k}, u \in W^{1, m}\left(\Omega ; \mathbb{R}^{m}\right)$ is necessary to make non ambiguous the definition of the integral functional. The conclusion (1.5) makes possible to extend the integral functional $d(u)$ in (1.2), a-priori uniquely defined in $W^{1, m}\left(\Omega ; \mathbb{R}^{m}\right)$, to functions in $W^{1, p}\left(\Omega ; \mathbb{R}^{m}\right)$ for $p>m-1$. See details for instance in [24].

We emphasize that in fact in this paper we give some lower semicontinuity results under more general assumptions than those stated above (see details in Theorems 3.6 and 4.5). Relevant aspects, in particular, are: (i) we deal with integrands $f$ depending on $(x, u)$ and on all the minors of the matrix $D u$; (ii) coercivity of $f$ is not assumed; (iii) we consider the weak convergence in $W^{1, p}\left(\Omega, \mathbb{R}^{m}\right)$ for $p>m-1$; i.e., below the natural exponent $p=m$ which guarantee a non ambiguous definition of the integrals. We also notice that our results hold in the limit case $p=m-1=1$, if $m=n=2$ (see Proposition 3.7).

The proof of the theorem stated above is based on an interesting combination of two principal ingredients, which reflect the basic role respectively of the convexity of $f$ and the polyconvexity of $g$ in (1.4). The first one is the original approximation argument for a convex function $f(x, s, \cdot)$ introduced by De Giorgi in [12]. In these notes $f$ is approximated from below by a sequence of linear functions whose coefficients, which depend on $(x, s)$, are explicitly represented in terms of $f$, allowing us to deduce regularity properties of the coefficients from the corresponding ones of $f$.

The second ingredient is a chain rule formula for determinants (see Lemmas 2.1 and 2.3 in Section 2). In the smooth case, for $m=n$, the chain rule has the following form: let $B=B(x, s)$ be a function of class $C^{1}$ defined in $\Omega \times \mathbb{R}^{n}$, and let $u \in W^{1, n}\left(\Omega, \mathbb{R}^{n}\right)$; under the notations

$$
\left\{\begin{array}{l}
v(x)=B(x, u(x)) \\
b(x, s)=\partial B / \partial s_{1} \\
D_{x} B(x, s)=\left(\frac{\partial B}{\partial x_{1}}, \frac{\partial B}{\partial x_{2}}, \ldots, \frac{\partial B}{\partial x_{n}}\right)
\end{array}\right.
$$

with $x \equiv\left(x_{i}\right)_{i=1, \ldots, n}$ and $s \equiv\left(s_{i}\right)_{i=1, \ldots, n}$, we have

$$
\operatorname{det}\left(D v, D u^{2}, \ldots, D u^{n}\right)=b(x, u) \operatorname{det} D u+\operatorname{det}\left(D_{x} B(x, u), D u^{2}, \ldots, D u^{n}\right),
$$

where $\left(D v, D u^{2}, \ldots, D u^{n}\right)$ represents the $n \times n$ matrix whose columns are the $n$ vectors $D v, D u^{2}, \ldots, D u^{n}$ and similarly for the other matrix $\left(D_{x} B(x, u), D u^{2}, \ldots\right.$, 
$D u^{n}$ ). The role and properties of determinants are crucial here. This formula (which admits a proper generalization to the case $m<n$ ) allows us to represent a term of the type $b(x, u)$ det $D u$ as an algebraic sum of Jacobian determinants, which are continuous in the weak topology of $W^{1, p}\left(\Omega, \mathbb{R}^{n}\right)$ for $p>n-1$. This allows us to pass to the limit as $k \rightarrow+\infty$ in expressions of the type

$$
\lim _{k \rightarrow+\infty} \int_{\Omega} b\left(x, u_{k}\right) \operatorname{det} D u_{k} d x=\int_{\Omega} b(x, u) \operatorname{det} D u d x .
$$

Let us finally mention that some recent lower semicontinuity results have been obtained, in the scalar case, using a chain rule formula for gradients (see $[19,18,9,10$, 20], see also [21] for convex functionals in the vector-valued case).

\section{Chain rule formulas for determinants}

In this section we study some chain rule formulas involving determinants. The first one is a pointwise formula and it is proved in a regular context, from which we derive an integral form under weaker assumptions.

In the following for a matrix $\xi=\left(\xi_{i}^{\alpha}\right)_{i=1,2, \ldots, n}^{\alpha=1,2, \ldots, n}$ we also use the notation det $\xi=$ $\operatorname{det}\left(\xi^{1}, \xi^{2}, \ldots, \xi^{n}\right)$, where, for $\alpha=1,2, \ldots, n$, the vector $\xi^{\alpha}=\left(\xi_{1}^{\alpha}, \xi_{2}^{\alpha}, \ldots, \xi_{n}^{\alpha}\right)$.

Lemma 2.1 (Pointwise chain rule). Let $B=B(x, s)$ be a function belonging to $e^{1}\left(\Omega \times \mathbb{R}^{n}\right)$. Then, for every $u \in W^{1, n}\left(\Omega ; \mathbb{R}^{n}\right) \cap L_{\mathrm{loc}}^{\infty}\left(\Omega ; \mathbb{R}^{n}\right)$, the function $v$ : $\Omega \rightarrow \mathbb{R}$, defined by

$$
v(x):=B(x, u(x)) \quad \text { for a.e. } x \in \Omega
$$

belongs to $W_{\mathrm{loc}}^{1, n}(\Omega)$ and, for almost every $x \in \Omega$, we have

$$
\begin{aligned}
D_{s_{1}} B\left(x, u^{1}(x), \ldots, u^{n}(x)\right) \operatorname{det} D u(x) & \\
= & \operatorname{det}\left(D v(x), D u^{2}(x), \ldots, D u^{n}(x)\right) \\
& \quad-\operatorname{det}\left(D_{x} B(x, u(x)), D u^{2}(x), \ldots, D u^{n}(x)\right) .
\end{aligned}
$$

Proof. Let $\Omega^{\prime} \subset \subset \Omega$. First of all we note that by the usual chain rule formula, for $j=1, \ldots, n$ and for almost every $x \in \Omega^{\prime}$, we obtain

$$
D_{x_{j}} v(x)=D_{x_{j}} B(x, u(x))+\sum_{i=1}^{n} D_{s_{i}} B(x, u(x)) D_{x_{j}} u^{i}(x) .
$$

Hence

$$
D v(x)=D_{x} B(x, u(x))+\sum_{i=1}^{n} D_{s_{i}} B(x, u(x)) D u^{i}(x) \quad \text { for a.e. } x \in \Omega^{\prime} .
$$


Therefore, by using the multilinearity properties of the determinants, it follows

$$
\begin{aligned}
\operatorname{det}\left(D v(x), D u^{2}(x), \ldots, D u^{n}(x)\right) \\
=\operatorname{det}\left(D_{x} B(x, u(x))+\sum_{i=1}^{n} D_{s_{i}} B(x, u(x)) D u^{i}(x), D u^{2}(x), \ldots, D u^{n}(x)\right) \\
=\operatorname{det}\left(D_{x} B(x, u(x)), D u^{2}(x), \ldots, D u^{n}(x)\right) \\
\quad+\sum_{i=1}^{n} D_{s_{i}} B(x, u(x)) \operatorname{det}\left(D u^{i}(x), D u^{2}(x), \ldots, D u^{n}(x)\right) .
\end{aligned}
$$

Since $\operatorname{det}\left(D u^{i}(x), D u^{2}(x), \ldots, D u^{n}(x)\right)=0$, for $i=2, \ldots, n,(2.1)$ follows for almost every $x \in \Omega^{\prime}$. Letting $\Omega^{\prime} \rightarrow \Omega$, the thesis is accomplished.

Remark 2.2. Formula (2.1) can be proved also for a general Borel function $B$ at every point $(x, s)$, with $s=u(x)$, in which $B$ is differentiable.

Moreover, we note that if $B=B(x, s)$ is a locally Lipschitz continuous function on $\Omega \times \mathbb{R}^{n}$, then equality (2.1) holds provided that $D_{s_{1}} B\left(x, u^{1}(x), \ldots, u^{n}(x)\right) \operatorname{det} D u(x)$ and $\operatorname{det}\left(D v(x), D u^{2}(x), \ldots, D u^{n}(x)\right)$ are interpreted to be zero whenever $D u(x)=$ 0 , irrespective of whether $D_{s_{1}} B\left(x, u^{1}(x), \ldots, u^{n}(x)\right)$ and $D v(x)$ are defined.

Finally, observe that (2.1) holds also with $s_{1}$ replaced with $s_{i}, i=1, \ldots, n$ :

$$
\begin{aligned}
D_{s_{i}} B(x, & \left.u^{1}(x), \ldots, u^{n}(x)\right) \operatorname{det} D u(x) \\
= & \operatorname{det}\left(D u^{1}(x), \ldots, D u^{i-1}(x), D v(x), D u^{i+1}(x), \ldots, D u^{n}(x)\right) \\
& \quad-\operatorname{det}\left(D u^{1}(x), \ldots, D u^{i-1}(x), D x B(x, u(x)), D u^{i+1}(x), \ldots, D u^{n}(x)\right) .
\end{aligned}
$$

Lemma 2.3 (Integral chain rule). Let $(x, s) \in \Omega \times \mathbb{R}^{n}$ and $s=\left(s_{1}, s^{\prime}\right) \in \mathbb{R} \times \mathbb{R}^{n-1}$. Let $B=B(x, s)$ be a continuous function such that, for every compact set $K \subset \Omega \times \mathbb{R}^{n}$ there exists a proper positive constant $C_{K}$, depending on $K$, such that

$$
\left|B\left(x, s_{1}, s^{\prime}\right)-B\left(y, t_{1}, s^{\prime}\right)\right| \leq C_{K}\left(|x-y|+\left|s_{1}-t_{1}\right|\right),
$$

for every $\left(x, s_{1}, s^{\prime}\right),\left(y, t_{1}, s^{\prime}\right) \in K$. Then, for every $u \in W^{1, n}\left(\Omega ; \mathbb{R}^{n}\right) \cap L_{\mathrm{loc}}^{\infty}\left(\Omega ; \mathbb{R}^{n}\right)$, we have

$$
\begin{aligned}
\int_{\Omega} \psi & D_{s_{1}} B\left(x, u^{1}, \ldots, u^{n}\right) \operatorname{det} D u d x \\
= & -\int_{\Omega} B(x, u) \operatorname{det}\left(D \psi, D u^{2}, \ldots, D u^{n}\right) d x \\
& -\int_{\Omega} \psi \operatorname{det}\left(D_{x} B(x, u), D u^{2}, \ldots, D u^{n}\right) d x
\end{aligned}
$$

for every $\psi \in \varphi_{0}^{1}(\Omega)$. 
Remark 2.4. We observe that, in the previous lemma, the assumption $u \in L_{\mathrm{loc}}^{\infty}\left(\Omega ; \mathbb{R}^{n}\right)$ can be removed if $B(x, s)=0$ for $|s| \geq L$, for some $L>0$.

Proof. Let $u \in W^{1, n}\left(\Omega ; \mathbb{R}^{n}\right) \cap L_{\mathrm{loc}}^{\infty}\left(\Omega ; \mathbb{R}^{n}\right)$, the function defined by

$$
\Omega \ni x \mapsto B(x, u(x))
$$

belongs to $W_{\text {loc }}^{1, n}(\Omega)$. Let $\Omega^{\prime} \subset \subset \Omega$ and $\rho_{\varepsilon}=\rho_{\varepsilon}\left(s^{\prime}\right)$ be a standard $\bigodot^{\infty}$-mollifier in $\mathbb{R}^{n-1}$. In what follows we will use the notation $B_{\varepsilon}$ to denote the convolution of $B$ with $\rho_{\varepsilon}$, i.e.

$$
B_{\varepsilon}(x, s)=B_{\varepsilon}\left(x, s_{1}, s^{\prime}\right)=\int_{\mathbb{R}^{n-1}} \rho_{\varepsilon}\left(s^{\prime}-\tau^{\prime}\right) B\left(x, s_{1}, \tau^{\prime}\right) d \tau^{\prime},
$$

for $x \in \Omega^{\prime}$ and $s \in \mathbb{R}^{n}$, where $0<\varepsilon<\operatorname{dist}\left(\Omega^{\prime}, \partial \Omega\right)$.

The function $v_{\varepsilon}(x)=B_{\varepsilon}(x, u(x))$ belongs to $W^{1, n}\left(\Omega^{\prime}\right)$ and, by multiplying (2.1) for every $\psi \in \mathcal{C}_{0}^{1}\left(\Omega^{\prime}\right)$ and integrating by parts, we obtain

$$
\begin{aligned}
\int_{\Omega} \psi & D_{s_{1}} B_{\varepsilon}\left(x, u^{1}, \ldots, u^{n}\right) \operatorname{det} D u d x \\
= & -\int_{\Omega} B_{\varepsilon}(x, u) \operatorname{det}\left(D \psi, D u^{2}, \ldots, D u^{n}\right) d x \\
& -\int_{\Omega} \psi \operatorname{det}\left(D_{x} B_{\varepsilon}(x, u), D u^{2}, \ldots, D u^{n}\right) d x
\end{aligned}
$$

Set $\|u\|_{\infty}=\|u\|_{L^{\infty}\left(\Omega^{\prime} ; \mathbb{R}^{m}\right)}$. We observe that, by assumptions, for a proper compact set $K \subset \Omega \times \mathbb{R}^{n}$ such that $\Omega^{\prime} \times B\left(0,\|u\|_{\infty}+1\right) \subset K$ and $(x, s) \in \operatorname{supp} \psi \times$ $B\left(0,\|u\|_{\infty}\right)$ and for every $\varepsilon>0$ we have

$$
\begin{aligned}
\left|D_{s_{1}} B_{\varepsilon}(x, s)\right| & \leq \int_{\mathbb{R}^{n-1}} \rho_{\varepsilon}\left(s^{\prime}-\tau^{\prime}\right)\left|D_{s_{1}} B\left(x, s_{1}, \tau^{\prime}\right)\right| d \tau^{\prime} \leq C_{K}, \\
\left|B_{\varepsilon}(x, s)\right| & \leq \int_{\mathbb{R}^{n-1}} \rho_{\varepsilon}\left(s^{\prime}-\tau^{\prime}\right)\left|B\left(x, s_{1}, \tau^{\prime}\right)\right| d \tau^{\prime} \leq C_{K}, \\
\left|D_{x} B_{\varepsilon}(x, s)\right| & \leq \int_{\mathbb{R}^{n-1}} \rho_{\varepsilon}\left(s^{\prime}-\tau^{\prime}\right)\left|D_{x} B_{\varepsilon}\left(x, s_{1}, \tau^{\prime}\right)\right| d \tau^{\prime} \leq C_{K},
\end{aligned}
$$

where we denote by $C_{K}$ also the positive constant such that $|B(x, s)| \leq C_{K}$, for $(x, s) \in K$.

The previous estimates and standard properties of convolution imply

$$
\begin{aligned}
D_{S_{1}} B_{\varepsilon} & \rightarrow D_{s_{1}} B & & \text { weakly* in } L^{\infty}\left(\operatorname{supp} \psi \times B\left(0,\|u\|_{\infty}+1\right)\right), \\
B_{\varepsilon} & \rightarrow B & & \text { weakly* in } L^{\infty}\left(\operatorname{supp} \psi \times B\left(0,\|u\|_{\infty}+1\right)\right), \\
D_{x} B_{\varepsilon} & \rightarrow D_{x} B & & \text { weakly* in } L^{\infty}\left(\operatorname{supp} \psi \times B\left(0,\|u\|_{\infty}\right)\right),
\end{aligned}
$$

and hence, since det $D u$ belongs to $L^{1}(\Omega)$, the thesis follows, once we pass to the limit for $\varepsilon \rightarrow 0^{+}$in (2.4). 


\section{Lower semicontinuity in the model case $m=n$}

In this section we prove the lower semicontinuity result in the model case $m=n$, when the function $g(x, s, \xi)$ appearing in the integral functional in (1.2) explicitly depends only on $(x, s)$ and the determinant $\operatorname{det} \xi=\operatorname{adj}_{n} \xi$ of the $n \times n$ matrix $\xi$; i.e., $g$ can be represented under the form

$$
g(x, s, \xi)=f(x, s, \operatorname{det} \xi),
$$

the general case being considered in the next section.

In the following we use two results, which are classical tools in the framework of lower semicontinuity problems. Firstly, we recall the approximation theorem for convex functions due to De Giorgi (see [12]). This result states that any convex function $f: \mathbb{R}^{v} \rightarrow \mathbb{R}, v \geq 1$, is approximated by mean of a sequence of affine functions $a_{j}+b_{j} \cdot \xi, a_{j} \in \mathbb{R}$ and $b_{j} \in \mathbb{R}^{v}$, where

$$
\begin{aligned}
a_{j} & :=\int_{\mathbb{R}^{v}} f(\eta)\left((v+1) \alpha_{j}(\eta)+\nabla \alpha_{j}(\eta) \cdot \eta\right) d \eta, \\
b_{j} & :=-\int_{\mathbb{R}^{v}} f(\eta) \nabla \alpha_{j}(\eta) d \eta,
\end{aligned}
$$

with $\alpha_{j} \in C_{0}^{1}\left(\mathbb{R}^{v}\right), j \in \mathbb{N}$, a nonnegative function such that $\int_{\mathbb{R}^{\nu}} \alpha_{j}(\eta) d \eta=1$.

Lemma 3.1. Let $f: \mathbb{R}^{v} \rightarrow \mathbb{R}$ be a convex function and $a_{j}$, $b_{j}$ be defined as in (3.1) and (3.2). Then the following property holds:

$$
f(\xi)=\sup _{j \in \mathbb{N}}\left[a_{j}+b_{j} \cdot \xi\right] \quad \forall \xi \in \mathbb{R}^{v} .
$$

Remark 3.2. The main feature of this approximation is that the coefficients $a_{j}$ and $b_{j}$ depend explicitly on $f$. In particular, when $f$ depends also on $(x, s)$ we have that (3.1) and (3.2) become

$$
\begin{aligned}
& a_{j}(x, s):=\int_{\mathbb{R}^{v}} f(x, s, \eta)\left((v+1) \alpha_{j}(\eta)+\nabla \alpha_{j}(\eta) \cdot \eta\right) d \eta, \\
& b_{j}(x, s):=-\int_{\mathbb{R}^{v}} f(x, s, \eta) \nabla \alpha_{j}(\eta) d \eta,
\end{aligned}
$$

which permit to deduce regularity properties with respect to $(x, s)$ of the coefficients $a_{j}, b_{j}$ from proper hypotheses satisfied by $f$. Hence, if $f$ satisfies some continuity or Lipschitz continuity assumptions with respect to $(x, s)$, then $a_{j}$ and $b_{j}$ inherit the same properties, too.

Now we recall the following localization lemma proven in [13]. 
Lemma 3.3. Let $\mu$ be a positive Radon measure defined on an open set $\Omega \subset \mathbb{R}^{n}$. Consider a sequence $\left\{\phi_{j}\right\}$ of Borel positive functions defined on $\Omega$. Then

$$
\int_{\Omega} \sup _{j \in \mathbb{N}} \phi_{j} d \mu=\sup _{j \in \mathbb{N}}\left(\sum_{i=1}^{j} \int_{A_{i}} \phi_{i} d \mu: A_{i} \subset \subset \Omega \text { open and pairwise disjoint }\right) \text {. }
$$

In order to approach our lower semicontinuity problem for polyconvex integrals, we firstly deal with functionals of the following type:

$$
u \in W^{1, n}\left(\Omega ; \mathbb{R}^{n}\right) \mapsto \int_{\Omega}[a(x, u)+b(x, u) \operatorname{det} D u]^{+} d x
$$

which, in view of the previous Lemma 3.1, will be the affine approximations of the general polyconvex functionals.

Lemma 3.4. Let $a=a(x, s)$ and $b=b(x, s)$ be two continuous functions. Assume that, for every compact set $K \subset \Omega \times \mathbb{R}^{n}$ and for every $(x, s),(y, s) \in K, b$ satisfies

$$
|b(x, s)-b(y, s)| \leq C_{K}|x-y|
$$

for a proper positive constant $C_{K}$, depending on the compact set $K$. Moreover we assume that $D_{x} b$ is a Carathéodory function. Then for every $u_{k}, u \in W^{1, n}\left(\Omega ; \mathbb{R}^{n}\right)$ with $u_{k} \rightarrow u$ weakly in $W^{1, p}\left(\Omega ; \mathbb{R}^{n}\right), p>n-1$, we have

$$
\int_{\Omega}[a(x, u)+b(x, u) \operatorname{det} D u]^{+} d x \leq \liminf _{k \rightarrow+\infty} \int_{\Omega}\left[a\left(x, u_{k}\right)+b\left(x, u_{k}\right) \operatorname{det} D u_{k}\right]^{+} d x \text {. }
$$

Proof. We split the proof in two steps.

Step 1: First we assume that $a(x, s)=0$ and $b(x, s)=0$ for $|s| \geq L$ for some $L>0$. By Lemma 3.3, it is easy to check that for every $u \in W^{1, n}\left(\Omega ; \mathbb{R}^{n}\right)$ we have

$$
\begin{aligned}
& \int_{\Omega} \psi {[a(x, u)+b(x, u) \operatorname{det} D u]^{+} d x } \\
&=\sup \left\{\int_{\Omega} \psi[a(x, u)+b(x, u) \operatorname{det} D u] d x: \psi \in C_{0}^{1}(\Omega), 0 \leq \psi \leq 1\right\} .
\end{aligned}
$$

Fix $\psi \in C_{0}^{1}(\Omega)$ with $0 \leq \psi \leq 1$ and let $K:=\operatorname{supp} \psi \times[-L, L]$. By Fatou's lemma, since $a$ is locally bounded, we obtain that

$$
\int_{\Omega} \psi a(x, u) d x \leq \liminf _{k \rightarrow \infty} \int_{\Omega} \psi a\left(x, u_{k}\right) d x .
$$

Define now

$$
B(x, s)=B\left(x, s_{1}, s^{\prime}\right):=\int_{0}^{s_{1}} b\left(x, \tau, s^{\prime}\right) d \tau
$$


so that $D_{s_{1}} B(x, s)=b(x, s)$. By applying Lemma 2.3 and Remark 2.4, it follows

$$
\begin{aligned}
\int_{\Omega} \psi & b\left(x, u_{k}\right) \operatorname{det} D u_{k} d x \\
= & \int_{\Omega} \psi D_{s_{1}} B\left(x, u_{k}^{1}, \ldots, u_{k}^{n}\right) \operatorname{det} D u_{k} d x \\
= & \int_{\Omega} B\left(x, u_{k}\right) \operatorname{det}\left(D \psi, D u_{k}^{2}, \ldots, D u_{k}^{n}\right) d x \\
& \quad-\int_{\Omega} \psi \operatorname{det}\left(D_{x} B\left(x, u_{k}\right), D u_{k}^{2}, \ldots, D u_{k}^{n}\right) d x
\end{aligned}
$$

Since $p>n-1$, for $q=\frac{p}{p-n+1}=\left(\frac{p}{n-1}\right)^{\prime}$, we have that

$$
\left\{\begin{array}{l}
B\left(x, u_{k}\right) \rightarrow B(x, u) \\
D_{x} B\left(x, u_{k}\right) \rightarrow D_{x} B(x, u)
\end{array} \quad \text { strongly in } L_{\mathrm{loc}}^{q}(\Omega),\right.
$$

and that all minors of order $n-1$ of the $n \times(n-1)$ matrix $\left(D u_{k}^{2}, \ldots, D u_{k}^{n}\right)$ weakly converges in $L^{\frac{p}{n-1}}(\Omega)$ (see [26]), therefore we get

$$
\begin{aligned}
\liminf _{k \rightarrow+\infty} \int_{\Omega} \psi b\left(x, u_{k}\right) \operatorname{det} D u_{k} d x \\
=-\int_{\Omega} B(x, u) \operatorname{det}\left(D \psi, D u^{2}, \ldots, D u^{n}\right) d x \\
\quad-\int_{\Omega} \psi \operatorname{det}\left(D_{x} B(x, u), D u^{2}, \ldots, D u^{n}\right) d x \\
=\int_{\Omega} \psi D_{s_{1}} B\left(x, u^{1}, \ldots, u^{n}\right) \operatorname{det} D u d x \\
=\int_{\Omega} \psi b(x, u) \operatorname{det} D u d x .
\end{aligned}
$$

By collecting (3.6) and (3.8) we obtain

$$
\begin{array}{r}
\liminf _{n \rightarrow \infty} \int_{\Omega} \psi\left[a\left(x, u_{k}\right)+b\left(x, u_{k}\right) \operatorname{det} D u_{k}\right] d x \\
\geq \int_{\Omega} \psi[a(x, u)+b(x, u) \operatorname{det} D u] d x
\end{array}
$$

and by taking the supremum over all $\psi$, we get the desired result.

Step 2: We now remove the extra assumption that $a(x, u)=0$ and $b(x, u)=0$ for $|u| \geq L$. 
For every $j \in \mathbb{N}$ define

$$
\sigma_{j}(s):= \begin{cases}1 & |s| \leq j-1 \\ -|s|+j & j-1<|s| \leq j, \\ 0 & |s|>j .\end{cases}
$$

Then, for every fixed $j \in \mathbb{N}$, we have

$$
\begin{aligned}
\liminf _{k \rightarrow \infty} & \int_{\Omega}\left[a\left(x, u_{k}\right)+b\left(x, u_{k}\right) \operatorname{det} D u_{k}\right]^{+} d x \\
& \geq \liminf _{k \rightarrow \infty} \int_{\Omega} \sigma_{j}\left(u_{k}\right)\left[a\left(x, u_{k}\right)+b\left(x, u_{k}\right) \operatorname{det} D u_{k}\right]^{+} d x .
\end{aligned}
$$

Since the functions

$$
a_{j}(x, s):=\sigma_{j}(s) a(x, s), \quad b_{j}(x, s):=\sigma_{j}(s) b(x, s)
$$

satisfy the hypotheses of Step 1, (3.9) and (3.11) imply

$$
\begin{aligned}
\liminf _{k \rightarrow \infty} & \int_{\Omega}\left[a\left(x, u_{k}\right)+b\left(x, u_{k}\right) \operatorname{det} D u_{k}\right]^{+} d x \\
& \geq \int_{\Omega} \sigma_{j}(u)[a(x, u)+b(x, u) \operatorname{det} D u]^{+} d x \\
& \geq \int_{\{x \in \Omega:|u(x)| \leq j-1\}}[a(x, u)+b(x, u) \operatorname{det} D u]^{+} d x
\end{aligned}
$$

Letting $j \rightarrow \infty$, the conclusion follows by Beppo Levi's lemma.

Remark 3.5. Note that, if $n=2$, the previous result continues to hold also in the limit case $p=n-1=1$. Indeed, following the idea of Dacorogna and Marcellini [7, Lemma 2], we have the equality

$$
\begin{aligned}
\lim _{k \rightarrow+\infty} & {\left[\int_{\Omega} B\left(x, u_{k}\right) \operatorname{det}\left(D \psi, D u_{k}^{2}\right) d x+\int_{\Omega} \psi \operatorname{det}\left(D_{x} B\left(x, u_{k}\right), D u_{k}^{2}\right) d x\right] } \\
& =\int_{\Omega} B(x, u) \operatorname{det}\left(D \psi, D u^{2}\right) d x+\int_{\Omega} \psi \operatorname{det}\left(D_{x} B(x, u), D u^{2}\right) d x
\end{aligned}
$$

and then (3.8) still holds true for $u_{k}$ converging to $u$ weakly in $W^{1,1}\left(\Omega ; \mathbb{R}^{2}\right)$.

Now we are in the position to prove a lower semicontinuity inequality for polyconvex integrals of the form

$$
\int_{\Omega} f(x, u, \operatorname{det} D u) d x
$$

along sequences $\left\{u_{k}\right\} \subset W^{1, n}\left(\Omega ; \mathbb{R}^{n}\right)$ weakly converging in $W^{1, p}\left(\Omega ; \mathbb{R}^{n}\right)$, for $p>$ $n-1$, without requiring any growth conditions on the integrand $f$. 
Theorem 3.6. Let $f: \Omega \times \mathbb{R}^{n} \times \mathbb{R} \rightarrow[0,+\infty)$ be a continuous function such that

for a.e. $x \in \Omega$ and for all $s \in \mathbb{R}^{n} \quad f(x, s, \cdot)$ is convex;

$\left\{\begin{array}{l}\forall K \subset \subset \Omega \times \mathbb{R}^{n} \times \mathbb{R} \quad \exists C_{K}>0 \text { such that } \\ |f(x, s, t)-f(y, s, t)| \leq C_{K}|x-y| \quad \forall(x, s, t),(y, s, t) \in K ;\end{array}\right.$

for every $(x, t) \in \Omega \times \mathbb{R} \quad D_{x} f(x, \cdot, t)$ is a continuous function .

Then for every $u_{k}, u \in W^{1, n}\left(\Omega ; \mathbb{R}^{n}\right)$ with $u_{k} \rightarrow u$ weakly in $W^{1, p}\left(\Omega ; \mathbb{R}^{n}\right), p>n-1$, we have

$$
\int_{\Omega} f(x, u, \operatorname{det} D u) d x \leq \liminf _{k \rightarrow \infty} \int_{\Omega} f\left(x, u_{k}, \operatorname{det} D u_{k}\right) d x .
$$

Proof. Let $\left\{u_{k}\right\}$ be a sequence in $W^{1, n}\left(\Omega ; \mathbb{R}^{n}\right)$ converging to $u \in W^{1, n}\left(\Omega ; \mathbb{R}^{n}\right)$, with respect to the $w-W^{1, p}$ convergence, with $p>n-1$. Since by Lemma 3.1 and Remark 3.2

$$
f(x, s, t)=\sup _{j \in \mathbb{N}}\left[a_{j}(x, s)+b_{j}(x, s) t\right]^{+},
$$

where $a_{j}$ and $b_{j}$ are defined as in (3.3) and (3.4), for every fixed $j \in \mathbb{N}$, we have

$$
\liminf _{k \rightarrow+\infty} \int_{\Omega} f\left(x, u_{k}, \operatorname{det} D u_{k}\right) d x \geq \liminf _{k \rightarrow+\infty} \int_{\Omega}\left[a_{j}\left(x, u_{k}\right)+b_{j}\left(x, u_{k}\right) \operatorname{det} D u_{k}\right]^{+} d x .
$$

By our assumptions, for every $j \in \mathbb{N}$ the functions $a_{j}$ and $b_{j}$ satisfy all the assumptions of Lemma 3.4. Therefore, we obtain

$$
\left.\liminf _{k \rightarrow+\infty} \int_{\Omega} f\left(x, u_{k}, \operatorname{det} D u_{k}\right) d x \geq \int_{\Omega}\left[a_{j}(x, u)+b_{j}(x, u) \operatorname{det} D u\right)\right]^{+} d x .
$$

Hence

$$
\liminf _{k \rightarrow+\infty} \int_{\Omega} f\left(x, u_{k}, \operatorname{det} D u_{k}\right) d x \geq \sup _{j \in \mathbb{N}} \int_{\Omega}\left[a_{j}(x, u)+b_{j}(x, u) \operatorname{det} D u\right]^{+} d x .
$$

The thesis follows by the localization Lemma 3.3.

We note that, as in [7], in the special case $n=2$ the previous result holds also in the limit case $p=n-1=1$, as stated in the following proposition, which can be proved by using Remark 3.5.

Theorem 3.7. Let $n=2$ and $p=1$. Assume that $\Omega \subset \mathbb{R}^{2}$ is an open set and $f: \Omega \times \mathbb{R}^{2} \times \mathbb{R} \rightarrow[0,+\infty)$ is a continuous function satisfying (3.12), (3.13) and (3.14). Then for every $u_{k}, u \in W^{1,2}\left(\Omega ; \mathbb{R}^{2}\right)$ with $u_{k} \rightarrow u$ weakly in $W^{1,1}\left(\Omega ; \mathbb{R}^{2}\right)$, we have

$$
\int_{\Omega} f(x, u, \operatorname{det} D u) d x \leq \liminf _{k \rightarrow+\infty} \int_{\Omega} f\left(x, u_{k}, \operatorname{det} D u_{k}\right) d x .
$$




\section{Lower semicontinuity in more general cases}

In this section we extend Theorem 3.6 to more general contexts.

\subsection{Dependence on all minors: the case $m=n$}

In this section we extend Theorem 3.6 to the case generally considered in the literature, where $f$ depends on all the minors of the Jacobian matrix $D u$.

Let

$$
\sigma=\sum_{k=1}^{n-1}\left(\begin{array}{l}
n \\
k
\end{array}\right)^{2}
$$

and for any matrix $\xi \in M I^{n \times n}$, we denote by $M^{n-1}(\xi)$ the vector in $\mathbb{R}^{\sigma}$, whose components are given by the determinants of all minors of $\xi$ up to the order $n-1$; i.e.,

$$
M(\xi)=\left(\xi, \operatorname{adj}_{2} \xi, \ldots, \operatorname{adj}_{i} \xi, \ldots, \operatorname{adj}_{n} \xi\right)=\left(M^{n-1}(\xi), \operatorname{det} \xi\right)
$$

As above, we firstly deal with functionals of the form:

$u \in W^{1, n}\left(\Omega ; \mathbb{R}^{n}\right) \mapsto \int_{\Omega}\left[a(x, u)+b^{\prime}(x, u) \cdot M^{n-1}(D u)+b_{\sigma+1}(x, u) \operatorname{det} D u\right]^{+} d x$,

where $a: \Omega \times \mathbb{R}^{n} \rightarrow \mathbb{R}$ and $b=\left(b^{\prime}, b_{\sigma+1}\right): \Omega \times \mathbb{R}^{n} \rightarrow \mathbb{R}^{\sigma} \times \mathbb{R}$.

Lemma 4.1. Let $a: \Omega \times \mathbb{R}^{n} \rightarrow \mathbb{R}$ and $b=\left(b^{\prime}, b_{\sigma+1}\right): \Omega \times \mathbb{R}^{n} \rightarrow \mathbb{R}^{\sigma} \times \mathbb{R}$ be continuous functions. Assume that, for every compact set $K \subset \Omega \times \mathbb{R}^{n}$ and for every $(x, s),(y, s) \in K, b_{\sigma+1}$ satisfy

$$
\left|b_{\sigma+1}(x, s)-b_{\sigma+1}(y, s)\right| \leq C_{K}|x-y|
$$

for a proper positive constant $C_{K}$, depending on the compact set $K$. Moreover we assume that $D_{x} b_{\sigma+1}$ is a Carathéodory function. Then for every $u_{k}, u \in W^{1, n}\left(\Omega ; \mathbb{R}^{n}\right)$ with $u_{k} \rightarrow u$ weakly in $W^{1, p}\left(\Omega ; \mathbb{R}^{n}\right), p>n-1$, we have

$$
\begin{array}{r}
\int_{\Omega}\left[a(x, u)+b^{\prime}(x, u) \cdot M^{n-1}(D u)+b_{\sigma+1}(x, u) \operatorname{det} D u\right]^{+} d x \\
\leq \liminf _{k \rightarrow+\infty} \int_{\Omega}\left[a\left(x, u_{k}\right)+b^{\prime}\left(x, u_{k}\right) \cdot M^{n-1}\left(D u_{k}\right)\right. \\
\left.+b_{\sigma+1}\left(x, u_{k}\right) \operatorname{det} D u_{k}\right]^{+} d x
\end{array}
$$

Proof. We can assume that $a(x, s)=0$ and $b(x, s)=0$ for $|s| \geq L$ for some $L>0$, since the general case follows by using the same truncation arguments as in the proof 
of Lemma 3.4. Recalling that for every $u \in W^{1, n}\left(\Omega ; \mathbb{R}^{n}\right)$ we have

$$
\begin{gathered}
\int_{\Omega}\left[a(x, u)+b^{\prime}(x, u) \cdot M^{n-1}(D u)+b_{\sigma+1}(x, u) \operatorname{det} D u\right]^{+} d x \\
=\sup \left\{\int_{\Omega} \psi\left[a(x, u)+b^{\prime}(x, u) \cdot M^{n-1}(D u)+b_{\sigma+1}(x, u) \operatorname{det} D u\right] d x:\right. \\
\left.\psi \in C_{0}^{1}(\Omega), 0 \leq \psi \leq 1\right\},
\end{gathered}
$$

and taking into account the localization Lemma 3.3, in order to achieve the conclusion, it is enough to prove that for all $\psi \in \mathcal{C}_{0}^{1}(\Omega)$ :

$$
\begin{aligned}
& \int_{\Omega} \psi\left[a(x, u)+b^{\prime}(x, u) \cdot M^{n-1}(D u)+b_{\sigma+1}(x, u) \operatorname{det} D u\right] d x \\
& \quad \leq \liminf _{k \rightarrow+\infty} \int_{\Omega} \psi\left[a\left(x, u_{k}\right)+b^{\prime}\left(x, u_{k}\right) \cdot M^{n-1}\left(D u_{k}\right)+b_{\sigma+} 1\left(x, u_{k}\right) \operatorname{det} D u_{k}\right] d x
\end{aligned}
$$

for $u_{k}, u \in W^{1, n}\left(\Omega ; \mathbb{R}^{n}\right)$ with $u_{k} \rightarrow u$ weakly in $W^{1, p}\left(\Omega ; \mathbb{R}^{n}\right)$.

By proceeding as in the proof of Lemma 3.4, since (3.9) holds with $b$ replaced by $b_{\sigma+1}$, we have only to check that

$$
\lim _{k \rightarrow+\infty} \int_{\Omega} \psi b^{\prime}\left(x, u_{k}\right) \cdot M^{n-1}\left(D u_{k}\right) d x=\int_{\Omega} \psi b^{\prime}(x, u) \cdot M^{n-1}(D u) d x .
$$

Formula (4.3) is a direct consequence of the fact that $b^{\prime}\left(x, u_{k}\right) \rightarrow b^{\prime}(x, u)$ strongly in $L^{q}\left(\Omega, \mathbb{R}^{\sigma}\right)$, for $q=\frac{p}{p-n+1}=\left(\frac{p}{n-1}\right)^{\prime}$ and all minors up to order $n-1$ of the $n \times n$ matrix $D u_{k}$ weakly converges in $L^{\frac{p}{n-1}}(\Omega)$ (see [26]).

Theorem 4.2. Let $f: \Omega \times \mathbb{R}^{n} \times \mathbb{R}^{\sigma} \times \mathbb{R} \rightarrow[0,+\infty)$ be a continuous function such that

for a.e. $x \in \Omega$ and for all $s \in \mathbb{R}^{n} \quad f(x, s, \cdot, \cdot)$ is convex;

$$
\begin{aligned}
& \left\{\begin{array}{l}
\forall K \subset \subset \Omega \times \mathbb{R}^{n} \times \mathbb{R}^{\sigma} \times \mathbb{R} \quad \exists C_{K}>0 \text { such that } \\
|f(x, s, \eta, t)-f(y, s, \eta, t)| \leq C_{K}|x-y| \quad \forall(x, s, \eta, t),(y, s, \eta, t) \in K ;
\end{array}\right. \\
& \forall(x, \eta, t) \in \Omega \times \mathbb{R}^{\sigma} \times \mathbb{R} \quad D_{x} f(x, \cdot, \eta, t) \text { is a continuous function. }
\end{aligned}
$$

Then for every $u_{k}, u \in W^{1, n}\left(\Omega ; \mathbb{R}^{n}\right)$ with $u_{k} \rightarrow u$ weakly in $W^{1, p}\left(\Omega ; \mathbb{R}^{n}\right), p>n-1$, we have

$$
\begin{aligned}
& \int_{\Omega} f\left(x, u, M^{n-1}(D u), \operatorname{det} D u\right) d x \\
& \quad \leq \liminf _{k \rightarrow+\infty} \int_{\Omega} f\left(x, u_{k}, M^{n-1}\left(D u_{k}\right), \operatorname{det} D u_{k}\right) d x .
\end{aligned}
$$


Proof. Let $\left\{u_{k}\right\}$ be a sequence in $W^{1, n}\left(\Omega ; \mathbb{R}^{n}\right)$ converging to $u \in W^{1, n}\left(\Omega ; \mathbb{R}^{n}\right)$, with respect to the $w-W^{1, p}$ convergence. By Lemma 3.1 with $v=\sigma+1$ and $\xi=(\eta, t)$, we have

$$
f(x, s, \eta, t)=\sup _{j \in \mathbb{N}}\left[a_{j}(x, s)+\left(b^{\prime}\right)_{j}(x, s) \cdot \eta+\left(b_{\sigma+1}\right)_{j}(x, s) t\right]^{+}
$$

where, for every $j \in \mathbb{N}, a_{j}$ and $b_{j}=\left(\left(b^{\prime}\right)_{j},\left(b_{\sigma+1}\right)_{j}\right) \in \mathbb{R}^{\sigma} \times \mathbb{R}$ are defined as in (3.3) and (3.4). Hence, for every $j \in \mathbb{N}$, we have

$$
\begin{aligned}
\liminf _{k \rightarrow+\infty} \int_{\Omega} f\left(x, u_{k}, M^{n-1}\left(D u_{k}\right), \operatorname{det} D u_{k}\right) d x \\
\geq \liminf _{k \rightarrow+\infty} \int_{\Omega}\left[a_{j}\left(x, u_{k}\right)+\left(b^{\prime}\right)_{j}\left(x, u_{k}\right) \cdot M^{n-1}\left(D u_{k}\right)\right. \\
\left.\quad+\left(b_{\sigma+1}\right)_{j}\left(x, u_{k}\right) \operatorname{det} D u_{k}\right]^{+} d x .
\end{aligned}
$$

Since for every fixed $j \in \mathbb{N}$ the functions $a_{j}$ and $b_{j}$ satisfy the assumptions of Lemma 4.1, we get that inequality (4.2) holds and the conclusion follows by proceeding as in the proof of Theorem 3.6.

\subsection{Dependence on all minors: the case $m<n$}

In this subsection we assume $u: \Omega \subseteq \mathbb{R}^{n} \rightarrow \mathbb{R}^{m}$ with $m<n$ and set

$$
\sigma^{\prime}=\sum_{j=1}^{m-1}\left(\begin{array}{l}
n \\
j
\end{array}\right)\left(\begin{array}{c}
m \\
j
\end{array}\right) \quad \text { and } \quad d=\left(\begin{array}{l}
n \\
m
\end{array}\right)
$$

For any matrix $\xi \in M I^{m \times n}$, we denote by $M^{m-1}(\xi)$ the vector in $\mathbb{R}^{\sigma^{\prime}}$, whose components are given by the determinants of all minors of $\xi$ up to the order $m-1$ and by $\operatorname{adj}_{m} \xi$ the vector in $\mathbb{R}^{d}$, whose components are given by the determinants of all minors of $\xi$ of the maximum order $m$.

Theorem 4.3. Let $f: \Omega \times \mathbb{R}^{m} \times \mathbb{R}^{\sigma^{\prime}} \times \mathbb{R}^{d} \rightarrow[0,+\infty)$ be a continuous function such that

for a.e. $x \in \Omega$ and for all $s \in \mathbb{R}^{m} \quad f(x, s, \cdot, \cdot)$ is convex;

$$
\begin{aligned}
& \left\{\begin{array}{l}
\forall K \subset \subset \Omega \times \mathbb{R}^{m} \times \mathbb{R}^{\sigma^{\prime}} \times \mathbb{R}^{d} \quad \exists C_{K}>0 \text { such that } \\
|f(x, s, \eta, \zeta)-f(y, s, \eta, \zeta)| \leq C_{K}|x-y| \quad \forall(x, s, \eta, \zeta),(y, s, \eta, \zeta) \in K
\end{array}\right. \\
& \forall(x, \eta, \zeta) \in \Omega \times \mathbb{R}^{\sigma^{\prime}} \times \mathbb{R}^{d}, D_{x} f(x, \cdot, \eta, \zeta) \text { is a continuous function } .
\end{aligned}
$$


Then for every $u_{k}, u \in W^{1, m}\left(\Omega ; \mathbb{R}^{m}\right)$ with $u_{k} \rightarrow u$ weakly in $W^{1, p}\left(\Omega ; \mathbb{R}^{m}\right), p>$ $m-1$, we have

$$
\begin{aligned}
& \int_{\Omega} f\left(x, u, M^{m-1}(D u), \operatorname{adj}_{m} D u\right) d x \\
& \quad \leq \liminf _{k \rightarrow+\infty} \int_{\Omega} f\left(x, u_{k}, M^{m-1}\left(D u_{k}\right), \operatorname{adj}_{m} D u_{k}\right) d x .
\end{aligned}
$$

Proof. Let $\left\{u_{k}\right\}$ be a sequence in $W^{1, m}\left(\Omega ; \mathbb{R}^{m}\right)$ converging to $u \in W^{1, m}\left(\Omega ; \mathbb{R}^{m}\right)$, with respect to the $w-W^{1, p}$ convergence, $p>m-1$. By Lemma 3.1, here $v=\sigma^{\prime}+d$ and $\xi=(\eta, \zeta)$, we have that

$$
f(x, s, \eta, \zeta)=\sup _{j \in \mathbb{N}}\left[a_{j}(x, s)+b_{j}^{\prime}(x, s) \cdot \eta+\bar{b}_{j}(x, s) \cdot \zeta\right]^{+}
$$

where, for every $j \in \mathbb{N}, a_{j}$ and $b_{j}=\left(b_{j}^{\prime},\left(b_{\sigma+1}\right)_{j}, \ldots,\left(b_{\sigma+d}\right)_{j}\right)=\left(b_{j}^{\prime}, \bar{b}_{j}\right) \in$ $\mathbb{R}^{\sigma^{\prime}} \times \mathbb{R}^{d}$ are defined in (3.3) and (3.4). Hence, for every fixed $j \in \mathbb{N}$, we have

$$
\begin{aligned}
& \liminf _{k \rightarrow+\infty} \int_{\Omega} f\left(x, u_{k}, M^{m-1}\left(D u_{k}\right), \operatorname{adj}_{m} D u_{k}\right) d x \\
& \geq \liminf _{k \rightarrow+\infty} \int_{\Omega}\left[a_{j}\left(x, u_{k}\right)+b_{j}^{\prime}\left(x, u_{k}\right) \cdot M^{m-1}\left(D u_{k}\right)+\bar{b}_{j}\left(x, u_{k}\right) \cdot \operatorname{adj}_{m} D u_{k}\right]^{+} d x .
\end{aligned}
$$

As in Lemma 3.4 we can assume that $a_{j}(x, s)=0$ and $b_{j}(x, s)=0$ for $|s| \geq L$ for some $L>0$, since the general case follows by the same truncation argument. Moreover, we recall that for every $j \in \mathbb{N}$ and every $u \in W^{1, m}\left(\Omega ; \mathbb{R}^{m}\right)$ we have

$$
\begin{gathered}
\int_{\Omega}\left[a_{j}(x, u)+b_{j}^{\prime}(x, u) \cdot M^{m-1}(D u)+\bar{b}_{j}(x, u) \cdot \operatorname{adj}_{m} D u\right]^{+} d x \\
=\sup \left\{\int_{\Omega} \psi\left[a_{j}(x, u)+b_{j}^{\prime}(x, u) \cdot M^{m-1}(D u)+\bar{b}_{j}(x, u) \cdot \operatorname{adj}_{m} D u\right] d x:\right. \\
\left.\psi \in C_{0}^{1}(\Omega), 0 \leq \psi \leq 1\right\} .
\end{gathered}
$$

Taking into account the localization Lemma 3.3, in order to achieve the thesis, it is enough to prove that for every $u_{k}, u \in W^{1, m}\left(\Omega ; \mathbb{R}^{m}\right)$ with $u_{k} \rightarrow u$ weakly in $W^{1, p}\left(\Omega ; \mathbb{R}^{m}\right)$,

$$
\begin{aligned}
& \int_{\Omega} \psi\left[a(x, u)+b^{\prime}(x, u) \cdot M^{m-1}(D u)+\bar{b}(x, u) \cdot \operatorname{adj}_{m} D u\right] d x \\
& \quad \leq \liminf _{k \rightarrow+\infty} \int_{\Omega} \psi\left[a\left(x, u_{k}\right)+b^{\prime}\left(x, u_{k}\right) \cdot M^{m-1}\left(D u_{k}\right)+\bar{b}\left(x, u_{k}\right) \cdot \operatorname{adj}_{m} D u_{k}\right] d x
\end{aligned}
$$


where for the sake of simplicity we omitted the subscript index $j$. By proceeding as in Lemmas 3.4 and 4.1 we get that (3.6) holds and it is easy to check that (4.3) continues to be true with $M^{n-1}$ replaced by $M^{m-1}$. Hence, we have only to check that

$$
\begin{aligned}
\lim _{k \rightarrow+\infty} & \int_{\Omega} \psi \bar{b}\left(x, u_{k}\right) \cdot \operatorname{adj}_{m} D u_{k} d x \\
& =\sum_{i=1}^{d}\left[\lim _{k \rightarrow+\infty} \int_{\Omega} \psi b_{\sigma+i}\left(x, u_{k}\right)\left(\operatorname{adj}_{m} D u_{k}\right)_{i} d x\right] \\
& =\sum_{i=1}^{d} \int_{\Omega} \psi b_{\sigma+i}(x, u)\left(\operatorname{adj}_{m} D u\right)_{i} d x \\
& =\int_{\Omega} \psi \bar{b}(x, u) \cdot \operatorname{adj}_{m} D u d x
\end{aligned}
$$

where $\bar{b}=\left(b_{\sigma+1}, \ldots, b_{\sigma+d}\right) \in \mathbb{R}^{d}$. Formula (4.10) is a consequence of the chain rule formula applied to $\left(\operatorname{adj}_{m} D u\right)_{i}$, for every $i=1, \ldots, d$. Indeed, fixed $i=1, \ldots, d$ and define the $\mathcal{C}^{1}$-function

$$
\bar{B}_{i}(x, s)=\bar{B}_{i}\left(x, s_{1}, s^{\prime}\right)=\int_{0}^{s_{1}} b_{\sigma+i}\left(x, \tau, s^{\prime}\right) d \tau
$$

(here $\left.s^{\prime} \in \mathbb{R}^{m-1}\right)$ and for $u \in W^{1, m}\left(\Omega ; \mathbb{R}^{m}\right)$ let $\bar{v}_{i}(x)=\bar{B}_{i}(x, u(x))$. Taking into account that $m<n$, with a proof similar to that of Lemma 2.1, we obtain:

$$
\begin{aligned}
b_{\sigma+i}\left(x, u^{1}, \ldots, u^{m}\right)\left(\operatorname{adj}_{m} D u\right)_{i}= & D_{s_{1}} \bar{B}_{i}\left(x, u^{1}, \ldots, u^{m}\right)\left(\operatorname{adj}_{m} D u\right)_{i} \\
= & \left(\operatorname{adj}_{m}\left(D \bar{v}_{i}, D u^{2}, \ldots, D u^{m}\right)\right)_{i} \\
& -\left(\operatorname{adj}_{m}\left(D_{x} \bar{B}_{i}(x, u), D u^{2}, \ldots, D u^{m}\right)\right)_{i} .
\end{aligned}
$$

The corresponding integral formulation is given by

$$
\begin{aligned}
\int_{\Omega} \psi & D_{s_{1}} \bar{B}_{i}\left(x, u^{1}, \ldots, u^{n}\right)\left(\operatorname{adj}_{m} D u\right)_{i} d x \\
= & -\int_{\Omega} \bar{B}_{i}(x, u)\left(\operatorname{adj}_{m}\left(D \psi, D u^{2}, \ldots, D u^{n}\right)\right)_{i} d x \\
& -\int_{\Omega} \psi\left(\operatorname{adj}_{m}\left(D_{x} \bar{B}_{i}(x, u), D u^{2}, \ldots, D u^{n}\right)\right)_{i} d x,
\end{aligned}
$$

for every $\psi \in \ell_{0}^{1}(\Omega)$. Finally, taking into account (4.12) and the fact that $p>$ $m-1,(4.10)$ can be obtained reasoning as in the proof of Lemma 3.4 (see in particular (3.8)). 


\subsection{Weakening of the regularity of the integrand}

In this subsection we consider the case where the integrand admits a Sobolev dependence (with an exponent $q \gg 1$ ) with respect to the $x$-variable instead of the Lipschitz continuity condition. To this purpose, we firstly need a proper new chain rule, under a Sobolev type dependence. For the sake of simplicity, we consider only the model case $m=n$ and $g(x, s, \xi)=f(x, s, \operatorname{det} \xi)$.

Lemma 4.4. Let $p>n-1, q=\frac{p}{p-n+1}=\left(\frac{p}{n-1}\right)^{\prime}$ and $B=B(x, s)$ be a function such that

$B$ is a Carathéodory function s.t. $B \in L_{\mathrm{loc}}^{\infty}\left(\Omega \times \mathbb{R}^{n}\right)$;

$\left\{\begin{array}{l}\forall s \in \mathbb{R}^{n} B(\cdot, s) \in W^{1, q}(\Omega), D_{x} B \quad \text { is a Carathéodory function and } \\ \forall K \subset \subset \Omega \times \mathbb{R}^{n} \exists a_{K} \in L^{q}(\Omega) \text { s.t. for every } s \in \mathbb{R}^{n} \text { and a.e. } x \in \Omega \\ \left|D_{x} B(x, s)\right| \leq a_{K}(x)\end{array}\right.$

for a.e. $x \in \Omega \quad B(x, \cdot) \in \mathcal{C}^{0}\left(\mathbb{R}^{n}\right)$;

$\left\{\begin{array}{l}B\left(x, \cdot, s^{\prime}\right) \in \mathcal{C}^{1}(\mathbb{R}) \text { for a.e. } x \in \Omega \text { and for every } s^{\prime} \in \mathbb{R}^{n-1} \\ \text { and } D_{s_{1}} B \text { is a Carathéodory function s.t. } D_{s_{1}} B \in L_{\mathrm{loc}}^{\infty}\left(\Omega \times \mathbb{R}^{n}\right) .\end{array}\right.$

Then, the same conclusions of Lemma 2.3 hold.

Proof. It is sufficient to follows the arguments in the proof of Lemma 2.3, by remarking that assumption (4.14) permits to pass to the limit, as $\varepsilon \rightarrow 0^{+}$, in (2.4).

By using previous lemma we obtain the following lower semicontinuity theorem.

Theorem 4.5. Let $p>n-1, q=\frac{p}{p-n+1}=\left(\frac{p}{n-1}\right)^{\prime}$ and $f: \Omega \times \mathbb{R}^{n} \times \mathbb{R} \rightarrow[0,+\infty)$ be a Borel function belonging to $L_{\mathrm{loc}}^{\infty}\left(\Omega \times \mathbb{R}^{n} \times \mathbb{R}\right)$ such that

for a.e. $x \in \Omega$ and for all $s \in \mathbb{R}^{n} f(x, s, \cdot)$ is convex;

for a.e. $x \in \Omega$ and for all $t \in \mathbb{R} \quad f(x, \cdot, t) \in \mathcal{C}^{0}\left(\mathbb{R}^{n}\right)$;

$\left\{\begin{array}{l}\forall(s, t) \in \mathbb{R}^{n} \times \mathbb{R} \quad f(\cdot, s, t) \in W^{1, q}(\Omega) \text { and } \\ \forall K \subset \subset \Omega \times \mathbb{R}^{n} \times \mathbb{R} \exists a_{K} \in L^{q}(\Omega) \text { s.t. } \\ \text { for a.e. } x \in \Omega \text { and for every }(s, t) \in \mathbb{R}^{n} \times \mathbb{R}\left|D_{x} f(x, s, t)\right| \leq a_{K}(x) ;\end{array}\right.$

for a.e. $x \in \Omega$ and for every $t \in \mathbb{R} D_{x} f(x, \cdot, t)$ is a continuous function.

Then for every $u_{k}, u \in W^{1, n}\left(\Omega ; \mathbb{R}^{n}\right)$ with $u_{k} \rightarrow u$ weakly in $W^{1, p}\left(\Omega ; \mathbb{R}^{n}\right)$, we have

$$
\int_{\Omega} f(x, u, \operatorname{det} D u) d x \leq \liminf _{k \rightarrow+\infty} \int_{\Omega} f\left(x, u_{k}, \operatorname{det} D u_{k}\right) d x .
$$


Remark 4.6. Under the same assumptions of Theorem 4.5, when $n=2$, the result still holds in the limit case $p=1$, by proceeding as in Theorem 3.7.

Moreover, condition (4.20) can be dropped if

$$
f(x, s, t)=g\left(x, s_{1}, t\right) h\left(s_{2}, \ldots, s_{n}\right),
$$

with $h$ continuous and $g$ satisfying (4.17)-(4.19).

Acknowledgments. The authors are grateful to Giovanni Leoni for helpful discussions during his visit in July 2006 to the Department of Mathematics of the University of Florence, Italy.

\section{References}

[1] E. Acerbi, G. Buttazzo and N. Fusco, Semicontinuity and relaxation for integrals depending on vector valued functions, J. Math. Pures Appl. 62 (1983), 371-387.

[2] E. Acerbi and G. Dal Maso, New lower semicontinuity result for polyconvex integrals, Calc. Var. Partial Differential Equations 2 (1994), 329-371.

[3] M. Amar, V. De Cicco and N. Fusco, Lower semicontinuity and relaxation results in BV for integral functionals with BV integrands, ESAIM Control Optim. Calc. Var., published online DOI: 10.1051/cocv:2007061(2007).

[4] J. M. Ball, Convexity conditions and existence theorems in nonlinear elasticity, Arch. Ration. Mech. Anal. 63 (1977), 337-403.

[5] P. Celada and G. Dal Maso, Further remarks on the lower semicontinuity of polyconvex integrals, Ann. Inst. H. Poincaré, Anal. Non Linéaire 11 (1994), 661-691.

[6] B. Dacorogna, Direct Methods in the Calculus of Variations, Appl. Math. Sci. 78, Springer, Berlin, 1989.

[7] B. Dacorogna and P. Marcellini, Semicontinuité pour des intégrandes polyconvexes sans continuité des déterminants, C.R. Math. Acad. Sci. Paris 311 (1990), 393-396.

[8] G. Dal Maso and C. Sbordone, Weak lower semicontinuity of polyconvex integrals: a borderline case, Math. Z. 218 (1995), 603-609.

[9] V. De Cicco, N. Fusco and A. Verde, On $L^{1}$-lower semicontinuity in $B V(\Omega)$, J. Convex Anal. 12 (2005), 173-185.

[10] V. De Cicco, N. Fusco and A. Verde, A chain rule formula in $B V(\Omega)$ and its applications to lower semicontinuity, Calc. Var. Partial Differential Equations 28 (2007), 427-447.

[11] V. De Cicco and G. Leoni, A chain rule in $L^{1}(\operatorname{div} ; \Omega)$ and its applications to lower semicontinuity, Calc. Var. Partial Differential Equations 19 (2004), 23-51.

[12] E. De Giorgi, Teoremi di semicontinuità nel calcolo delle variazioni, Istituto Nazionale di Alta Matematica, Roma, 1968-1969. 
[13] E. De Giorgi, G. Buttazzo and G. Dal Maso, On the lower semicontinuity of certain integral functions, Atti Accad. Naz. Lincei Cl. Sci. Fis. Mat. Natur. Rend. Lincei (9) Mat. Appl. 74 (1983), 274-282.

[14] I. Fonseca and G. Leoni, Some remarks on lower semicontinuity, Indiana Univ. Math. J. 49 (2000), 617-635.

[15] N. Fusco, M. Gori and F. Maggi, A remark on Serrin's Theorem, NoDEA Nonlinear Differential Equations Appl. 28 (2007), 427-447.

[16] N. Fusco and J.E. Hutchinson, A direct proof for lower semicontinuity of polyconvex functionals, Manuscripta Math. 87 (1995), 35-50.

[17] W. Gangbo, On the weak lower semicontinuity of energies with polyconvex integrands, J. Math. Pures Appl. 73 (1994), 455-469.

[18] M. Gori, F. Maggi and P. Marcellini, On some sharp conditions for lower semicontinuity in $L^{1}$, Differ. Integral Equ. Appl. 16 (2003), 51-76.

[19] M. Gori and P. Marcellini, An extension of the Serrin's lower semicontinuity theorem, J. Convex Anal. 9 (2002), 475-502.

[20] D. Graziani, A new $L^{1}$-lower semicontinuity result, to appear in Boll. Unione Mat. Ital. Sez. B Artic. Ric. Mat. (8).

[21] D. Graziani, On the $L^{1}$-lower Semicontinuity and Relaxation in $B V\left(\Omega ; \mathbb{R}^{m}\right)$ for Integral Functionals with Discontinuous Integrand, Ph.D. Thesis, Univ. "La Sapienza", Roma, 2007.

[22] J. Maly, Weak lower semicontinuity of polyconvex integrals, Proc. Edinb. Math. Soc. (2) 123 (1993), 681-691.

[23] P. Marcellini, Approximation of quasiconvex functions and lower semicontinuity of multiple integrals, Manuscripta Math. 51 (1985), 1-28.

[24] P. Marcellini, On the definition and the lower semicontinuity of certain quasiconvex integrals, Ann. Inst. H. Poincaré Anal. Non Linéaire 3 (1986), 391-409.

[25] C. B. Morrey, Multiple Integrals in the Calculus of Variations, Springer, Berlin, 1966.

[26] Y. Reshetnyak, Stability theorems for mappings with bounded distortion, Sibirsk. Mat. Zh. 9 (1968), 667-684.

[27] J. Serrin, On the definition and properties of certain variational integrals, Trans. Amer. Math. Soc. 161 (1961), 139-167.

Received July 17, 2007; revised October 31, 2007.

\section{Author information}

Micol Amar, Dipartimento di Metodi e Modelli Matematici

Università di Roma "La Sapienza"

Via A. Scarpa 16, 00161 Roma, Italy.

E-mail: amar@dmmm.uniroma1.it 
Virginia De Cicco, Dipartimento di Metodi e Modelli Matematici Università di Roma "La Sapienza"

Via A. Scarpa 16, 00161 Roma, Italy.

E-mail: decicco@dmmm.uniroma1.it

Paolo Marcellini, Dipartimento di Matematica "U. Dini"

Università di Firenze

Viale Morgagni 67/A, 50134 Firenze, Italy.

E-mail: marcellini@math.unifi.it

Elvira Mascolo, Dipartimento di Matematica "U. Dini”"

Università di Firenze

Viale Morgagni 67/A, 50134 Firenze, Italy.

E-mail: elvira.mascolo@math.unifi.it 